\title{
RATIONAL REPRESENTATION OF MODULAR NUMBERS
}

\author{
Peter Hintenaus Vilmar Trevisan*
}

\begin{abstract}
We find conditions, restricting the size of the fractions, and present algorithms to obtain a rational number from a set of residues modulo relatively prime integers. We also discuss the nature and the number of solutions for the rational representation, introducing conditions for the existence and for the uniqueness.
\end{abstract}

\section{Resumo}

Restringindo o tamanho das frações, condições são estabelecidas c algoritmos são apresentados para que um número racional scja obtido a partir de um conjunto de resíduos módulo inteiros que são primos entre si. Também são discutidos a natureza e o número de soluçóes para a representaça racional, estabelecendo condiçoses para a existência e unicidade.

\section{Introduction}

In Symbolic and Algebraic Computation, a wide range of problems have been solved very efficiently via a modular approach. Many problems over the integers are mapped orto a prime field and once the image of the solution is known; it is recovered to a true solution for the original problem, by means of the chimese remainder or Hensel p-adic libing algorithm. Representative examples of this approach include polynomial factorization algorithms (see, for example, [3]), polynomial GCD computations (sce, for instance, [3]) and Gröbncr basis computation algorithms [8].

Some of these problems would be more efliciently solved over the rationals, and other problems arise more naturally in this field. Can we design an efficient

*Partially supported by CAPES, Brazil, under grant 0744/96-04 
modular method for the rationals? Given a rational number $r=u / v$ and a positive integer $a$ relatively prime to $v$, it is easy to find an integer $-a / 2 \leq$ $k \leq a / 2$, such that $r \equiv k(\bmod a)$. If we want to develop modular approaches for the rational field, we need to go in the other direction, that is, given a residue $k$ ( $\bmod a)$, car we compute a rational number $r=u / v$ such that $r \equiv$ $k(\bmod a) ?$

In 1981, P. Wang [9] introduced an algorithm for recomstructing a rational number from a modular image. Given integers $a, b>0$, Wang's algorithm finds, if it cxists, a pair of integers $\alpha, \beta$, satisfying

$$
\alpha \equiv \beta b(\bmod a)
$$

and

$$
0 \leq|\alpha|, \beta<\sqrt{a / 2}, \quad \beta \neq 0
$$

The algorithm is a modification of the extended Euclidean algorithm (for the greatest commom divisor). Wang also observed, in his original paper, that if such a rational representation exists and if, additionally, $\operatorname{gcd}(\alpha, \beta)=1$, then 1.he representiation is unique.

Such a representation, however, may not exist. As an example, modulo 15 , we have representations $0=0 / 1,1=1 / 1,2=2 / 1,7=-1 / 2$. It's worth noticing that $3,4,5$ and 6 are not rational images (modulo 15 ) of fractions with numerator and denominator between -2 and 2 .

If the bound $\sqrt{a / 2}$ is relaxed, then more rational numbers can be represented. For instance, if we allow rational fractions with denominator bigger than 2 (but smaller than 5) then, modulo 15 , we have $3=3 / 1,6=-3 / 2$. Uniqueness, however, may not be assured. For instance, $1=1 / 1=1 / 1(\bmod 15)$.

We study a more general situation in this paper; fixing an arbitrary bound $c$ for the denominator $\beta$ and, as a consequence, adjusting the size of the numerator $\alpha$ in such a way that the product $\alpha \beta$ is as large as the modulo $a$. The problem is also generalized for a rational reconstruction that simultaneously satisfy several modular relalions. 
In section 2, we discuss the existence of the rational representation in this new set of conditions. We discuss the nature of the solutions and make a conjecture about the number of solutions that are relatively prime.

In section 3, we restrict the problem so that we obtain unique representation of a modular number as a rational number. We point out, however, that we may not assure existence of solutions.

Algorithms for recovering the rational numbers are introduced in section 4. Wo discuss Wang's algorithm, modifying it to obtain the rational representation in this new situation. We finally point out several applications appearing in the literature that make use of rational reconstruction.

\section{The Existence}

The material of this section is inspired by the statement of lemma 3 given in [4]. We rectify here some mistakes that appear in the result, and generalize its content, adding an extra parameter.

Let $a_{i}, b_{i}$ be positive integers for $1 \leq i \leq n$, where $a_{i}$ and $a_{j}$ are relatively prime for $i \neq j$. Ieet $A=\prod_{k=1}^{n} a_{i}$. Given positive integers $c$ and $m$, with $1 \leq m \leq c$, comsider the following

Problem 1: Find integers $\alpha, \beta$, such that

$$
\begin{gathered}
1 \leq \beta \leq c \\
0 \leq|\alpha| \leq\left\lceil\frac{A m}{2 c}\right\rceil, \\
\beta b_{i} \equiv \alpha\left(\bmod a_{i}\right) \text { for } 1 \leq i \leq n .
\end{gathered}
$$

The followirg result holds.

Theorem 1. If $m \geq 2$, then there exists a pair $(\alpha, \beta)$ solving problem 1 .

Proof. First we prove theorem 1 for $n=1$. Let $a=a_{1}, b=b_{1}$. Without loss of generality we assume $m=2$. Consider all pairs $(\gamma, \delta)$ where $-\left\lceil\frac{a}{2 c}\right\rceil<\gamma \leq\left\lceil\frac{a}{2 c}\right\rceil$, $1 \leq \delta \leq c$. There are $2\left\lceil\frac{a}{2 c}\right\rceil c \geq 2 \frac{a}{2 c} c=a$ such pairs. Let us assume there is 
no pair $(\gamma, \delta)$ such that $a \mid(\gamma-\delta b)$. 'Then by the pigeon hole principle there are two different pairs $\left(\gamma_{1}, \delta_{1}\right)$ and $\left(\gamma_{2}, \delta_{2}\right)$ such that $\gamma_{1}-\delta_{1} b \equiv \gamma_{2}-\delta_{2} b(\bmod a)$, $\delta_{1}>\delta_{2}$, i.e

$$
a \mid\left(\gamma_{1}-\gamma_{2}\right)-\left(\delta_{1}-\delta_{2}\right) b
$$

What remains to be shown is that $0 \leq\left|\gamma_{1}-\gamma_{2}\right| \leq\left\lceil\frac{a}{c}\right\rceil$.

Without loss of gencrality we assume $\gamma_{1}>0, \gamma_{2}<0$. Wo know $\gamma_{1} \leq\left\lceil\frac{a}{2 c}\right\rceil<$ $\frac{a}{2 c}+1$. Furthermore, $\left|\gamma_{2}\right| \leq\left\lceil\frac{a}{2 c}\right\rceil-1<\frac{a}{2 c}$. Both $\gamma_{1}$ and $\gamma_{2}$ are integers, so $\left|\gamma_{1}-\gamma_{2}\right| \leq\left|\gamma_{1}\right|+\left|\gamma_{2}\right| \leq\left\lceil\frac{a}{r}\right\rceil$.

For $n>1$, we can compute a nonnegative integer $B<A$ such that $B \equiv b_{i}$ (mod $\left.a_{i}\right)$ for $1 \leq i \leq n$, using the Chinese Remainder Algorithm. Let $(\alpha, \beta)$ be a solution to $\alpha \equiv \beta B(\bmod A)$. Then $a_{i} \mid\left(\alpha-\beta b_{i}\right)$ for $1 \leq i \leq n$. So, theorem 1 is proved.

Note: A special case of this result appears in Sorensen [7] (lemma 2.2) for $n=1, m=2$ and $c=\sqrt{a}$.

We observe that the existence of solutions for problem 1 is not granted for $m=1$. As a counter example, take $a=27, b=4$ and $c=5$. There are no solutions $0<\beta \leq c, \quad|\alpha| \leq 3$ to the congruence $4 \beta \equiv \alpha(\bmod a)$.

In some applications, oblaining any solution to problem 1 is enough to benefit from the rational reconstruction technique. New integer GCD algorithms, for example, use the following reduction to improve efficiency. Given integers $u>v$, relativcly prime to $\alpha$, find integcrs $\alpha, \beta$ satisfying $\alpha u+\beta v \equiv 0(\bmod \alpha)$, with $|\alpha|,|\beta|<\sqrt{a}$ and replace $u$ by $u^{\prime}=|\alpha u+\beta v| / a$, whose size is smaller than $u$ (actually the size of $u$ is reduced by roughly $\log _{2}(a) / 2$ bits). Notice now that $\operatorname{gcd}(u, v)$ can be recovered from $\operatorname{gcd}\left(u^{\prime}, v\right)$ (see $\left.[\overline{7}, 11]\right)$. The operation $\alpha u+\beta v \equiv 0$ (mod a) is equivalent to recomstructing a rational number from the residue $r=u / v(\bmod a)$. In this case, the existence of multiple solutioms are of no importance, as long as the bound $\sqrt{a}$ is observed, guarantecing the size reduction.

When dealing with other applications, however; certain properties of the 
solutions are some times required, beyond its simple existence. For example, if $m=2, \quad a=12, \quad b=4$ and $c=3$, then the only solutions to $4 \beta \equiv$ $\alpha(\bmod 12)$ with the constraints $1 \leq \beta \leq 3$ and $-4 \leq \alpha \leq 4$ are the pairs $(\alpha, \beta)=(4,1),(-4,2),(0,3)$. As rational numbers, the second and third pairs do not represent solutions, for we can not say that $0 / 3 \equiv 4$ (mod 12) or that $-4 / 2 \equiv 1(\bmod 12)$ since 3 and 2 are not invertible modulo 12.41 so, $0 / 3=$ $0 \not \equiv 4(\bmod 12)$ and $-4 / 2=-2 \not \equiv 4(\bmod 12)$. The problem is that 1hese 1 wo pairs are not relatincly prime.

A relatively prime solution pair, that is, a solution $(\alpha, \beta)$ to problem 1 with $m=2$ and $\operatorname{gcd}(\alpha, \beta)=1$ does not always exist. As an example, notice that the only solutions to $7 \beta \equiv \alpha(\bmod 24)$ with the constraints $1 \leq \beta \leq 5$ and $|\alpha| \leq 5$ are $(-3,3)$ and $(4,4)$.

Another issue is the uniqueness of solutions. For $m \geq 2$, the above examples show that problem 1 has no chance of having a unique solution. If only the relatively prime pairs are sought, neither existence is attained (as example above shows), nor uniqueness. To see the this, observe that, $\operatorname{modulo} 24, b=5=5 / 1=$ $-4 / 4=1 / 5$. That is, there are 1 wo relatively prime solutions to problem 1 , for $a=24, b=5, c=5$ and $m=2$.

We believe to be true the following conjecture regarding the number of solutions $(\alpha, \beta)$ with $\operatorname{gcd}(\alpha, \beta)=1$.

Conjecture: Problem 1 has at most $m$ relatively prime solution pairs.

This result is proven for $m=1$ in the next section. In other words, to guarantee uniqueness of relatively prime solutions, we need to restrict the size of numcrator (as function of the denominator). The existence of solutions, however, is no longer assured.

\section{The Uniqueness}

Let $a_{i}, b_{i}$ be positive integers for $1 \leq i \leq n$, where $a_{i}$ and $a_{j}$ are relatively prime For $i \neq j$. Iet $A=\prod_{k=1}^{n} a_{i}$. Given a positive integer $c$, consider the followirng 
Problem 2: Find integers $\alpha, \beta$, such that

$$
\begin{gathered}
1 \leq \beta \leq c \\
-\frac{A}{2 c}<\alpha \leq \frac{A}{2 c} \\
\beta b_{i} \equiv \alpha \quad\left(\bmod a_{i}\right) \text { for } 1 \leq i \leq n . \\
\operatorname{gcd}(\alpha, \beta)=1
\end{gathered}
$$

The following result holds.

Theorem 2. If there is a pair $(\alpha, \beta)$ satisfying problem 2. then it is unique.

Proof. Let $\left(\alpha_{1}, \beta_{1}\right)$ and $\left(\alpha_{2}, \beta_{2}\right)$ be two solutions of problem 2. Then, the relation

$$
\alpha_{1} \beta_{2} \equiv \alpha_{2} \beta_{1}\left(\bmod a_{i}\right) \quad i=1, \ldots, n
$$

holds, which implies that

$$
\alpha_{1} \beta_{2} \equiv \alpha_{2}, \beta_{1}(\bmod A)
$$

that is, there exists an integer $k$

$$
\alpha_{1} \beta_{2}-\alpha_{2} \beta_{1}=k \Lambda
$$

On the other hand,

$$
\left|\alpha_{1} \beta_{2}-\alpha_{2} \beta_{1}\right| \leq\left|\alpha_{1} \beta_{2}\right|+\left|\alpha_{2} \beta_{1}\right| \leq A .
$$

The only possibility for equality above is when $\beta_{1}=\beta_{2}=c$ and $\alpha_{1}=-\alpha_{2}=$ $\pm A / 2 c$ and this is impossible for either $\alpha_{1}$ or $\alpha_{2}$ is sut of the range given by equation (7).

It follows that

$$
\left|\alpha_{1} \beta_{2}-\alpha_{2} \beta_{1}\right|=k: A<A,
$$


implying that $k=0$ and because the pairs are relatively prime, $\alpha_{1}=\alpha_{2}$ and $\beta_{1}=\beta_{2}$, which proves the result.

We now turn to the problem of recovering rational numbers by presenting algorithums for Problems 1 and 2.

\section{Recovering Rational Numbers}

Wang's original problem may be vicwed as a restriction of problem 2 with $c=\sqrt{A / 2}, n=1$. As observed by Wang, a modification on the Euclidean extended algorithm can be used to compute such pair $(\alpha, \beta)$. 'The difference between his algorithm and the Fuclidean is that it has a distinct stop condition.

Collins \& Fncarnación [2], using multiple-precision techniques for integer ged computations, presented a more elficient version of Wang's algorithm for ralional reconstruction.

Consider now finding an integer solution $(\alpha, \beta)$ to problem 1 . Because of the Chinese Remainder Theorem and observation at the end of theorem 1, it is sufficient to consider $n=1$. The algorithm RATCON of figure 1, a slight modilicalion of Wang's [9], may be used to lind $(\alpha, \beta)$, salisfying conditions (3), (4) and (5).

The algorithm differs from Wang's in the stopping condition (step 3.1), since i1 considers an adjustable bound for the denominalor and mumetalor, depending on two parameters $(c$ and $m$ ).

The correctness of the algorithm has becn proved in [10] and in [6] for the special case $c=\sqrt{a / 2}$ and $m=1$ in the sense that the solution (if there is any) to $\alpha \equiv \beta b(\bmod a)$, with constraints $\alpha<\frac{\alpha}{2 c}, \quad 0<\beta \leq c$ is generated by the algorithm. Both proofs can be extended for the general case presented here. Since we are using $m \geq 2$, theorem 1 assures the existence of solutions, so $\mathrm{R} \Lambda \mathrm{TCON}$ will return a pair $(\alpha, \beta)$ satisfying conditions (3). (4) and (5). 
$\operatorname{RATCON}(a, b, c, m)$

INPUT : Integers, $a, b, c$ and $m$, with $2 \leq m \leq c<a$.

OUTPUT: $\Lambda$ solution $(\alpha, \beta)$ for problem 1 .

0 . Let check: $=\left\lceil\frac{a * m}{2 * c}\right\rceil, i:=1$

1. Let $\left(\alpha_{0}, \alpha_{1}\right):=(a, b)$

2. Let $\left(\beta_{0}, \beta_{1}\right):=(0,1)$

3. While $\beta_{i} \leq c$ do

$$
\begin{aligned}
& 3.1 \text { if }\left|\alpha_{i}\right|<=\text { check then return }\left(\operatorname{sign}\left(\beta_{i}\right) \alpha_{i},\left|\beta_{i}\right|\right) ; \\
& 3.2 q_{i}:=\left\lfloor\alpha_{i-1} / \alpha_{i}\right\rfloor \\
& 3.3 \alpha_{i+1}:=\alpha_{i-1}-q_{i} \alpha_{i} ; \\
& 3.1 \beta_{i+1}:=\beta_{i-1}-q_{i} \beta_{i} \\
& 3.5 i:=i+1
\end{aligned}
$$

Figure 1: Algorithm lor computing a solution to Problem 1

Let us now examine the output $(\alpha, \beta)$ of algorithm RA'l'CON. As the examples of the previous section show, we can say neither that it is the unique solution to problem 1 nor that $\operatorname{gcd}(\alpha, \beta)=1$.

Theorem 3. Let $(\alpha, \beta)$ be the oulpul of algorithm RATCON. Then the following properties are satisfied.

1. If an inleger $k$ divides $\operatorname{gcd}(\alpha, \beta)$ then $k$ divides $a$.

2. $\operatorname{gcd}(\alpha, \beta)=\operatorname{gcd}(\alpha, \beta)$.

\section{Proof.}

Property 2 is given in [2]. Clearly, property 1 may be secn as a conscquence of of property 2. However, it is also a consequence of the following loop invariant [11], that is interesting by itself.

$$
\alpha_{i} \beta_{i+1}-\alpha_{i+1} \beta_{i}=(-1)^{i} a, \quad \text { for } i=0,1, \ldots
$$

This can be easily proven by induction on $i$. Now, since $(\alpha, \beta)=\left(\alpha_{i+1}, \beta_{i+1}\right)$ 
for some $i$ and $k$ divides both $\alpha_{i+1}$ and $\beta_{i+1}$, it follows from (10) that $k$ divides $a$.

It is worth noticing that algorithm RAICON would not be able to generate all solutions to problem 1, even if we let stop condition (step 3.1 ) go to zero. For example, consider the congruence $8,3 \equiv 8(\bmod 27)$. The only possible outputs returned by the algorithm (depending on the stop comdition) are $(8,1),(-3,3),(2,7)$ and $(-1,10)$. The pair $(5,4)$, a solution to problem 1 with parameters $a=27, b=8, c=4$ and $m=2$, is not returned, and may be considered "better" then the non-relatively prime pair $(-3,3)$, the output of the algorithm ran with these parameters.

Consider now finding a solution to problem 2. The obvious use of algorithm RATCON with $m=1$ is not enough, since there could be no solutioms. An additional problem is that there is at most one satisfying $\operatorname{ged}(\alpha, \beta)=1$ (theorem 2). but there may cxist solutions that are non-relatively prime and if the algorithm RATCON above is used with $m=1$, it could return such a solution.

The first difficulty is easily fixed, adding an extra stopping condition. The following modification, introduced in [2], corrects the second problem. As above, we consider $n=1$. I'he algorithm of figure 2 finds, when it exists, the solution to problem 2.

The extra comditiom $\operatorname{gcd}(\alpha, \beta)=1$ al step 3.2 guatantees the co-primeness of $\alpha$ and $\beta$ and is equivalent to saying that $\beta$ is invertible in the ring of integers modulo $a$; in other words, that the rational $\frac{\alpha}{\beta}$ is well defined or that

$$
\frac{\alpha}{\beta}=b(\bmod a)
$$

\section{Applications}

The problems usually dealt in computer algebra that use rational reconstruction are polynomial factorization, partial fraction decomposition and Gröbner basis determination. It is feasible to use rational reconstruction for isolating polynomial roots. Actually, in [5], there is a modular version of the Sturm the- 
RATCONUN $(a, b, c)$

INPUT : Integer's, $a, b$ and $c, c<a$.

OUTPUT: $\Lambda$ solution $(\alpha, \beta)$ for problem 2 , if it exists, NIL otherwise.

1. Iset $\left(\alpha_{0}, \alpha_{1}\right):=(a, b)$;

2. Tet $\left(\beta_{0}, \beta_{1}\right):=(0,1)$;

3. For $i=1,2, \ldots$, do

3.1 if $\left|\beta_{i}\right|>c$ then return NIL;

3.2 if $\left|\alpha_{i}\right| \leq a / 2 c$ then if $\operatorname{gcd}\left(\alpha_{i}, \beta_{i}\right)=1$ then return $\left(\operatorname{sign}\left(\beta_{i}\right) \alpha_{i},\left|\beta_{i}\right|\right)$;

$3.3 q_{i}:=\left\lfloor\alpha_{i-1} / \alpha_{i}\right\rfloor$

$3.4 \alpha_{i+1}:=\alpha_{i-1}-q_{i} \alpha_{i}$

$3.5 \beta_{i+1}:=\beta_{i-1}-q_{i} \beta_{i}$

Figure 2: Algorithm for computing the solution to Problem 2

orem that counts the number of zeros of a real polynomial in an interval with rational endpoints.

As explained in section 2, rational reconstruction has also been used for reducing the input size in the computation of integer ged. It should be noted that, in this case, the inputs for the rational reconstruction are small, since it is itsell a ged computation.

In other applicalions where large inpuls are needed, the multi precision arithmetic algorithm given in [2] is recommended. Special care should be given 1.o the extra condition $\operatorname{gcd}(\alpha, \beta)=1$, since the mumbers $\alpha$ and $\beta$ can also be large. Most applications require working modulo a product of prime numbers (or prime powers), therefore the modulo $a$ has a known form and, because of 1.heorem 3, it is possible to efliciently undertake the step. Collins \& Frarartación [2] discuss ways to avoid or at least improve the efficiency for computing this condition.

Most applications look for the unique solution in the rational reconstruction problem. Some problems, like polynomial factorization, have a trial step at the end so it may be possible to use the algorithm RATCON, that is to say to find a 
solution to our problem 1 , which would be more efficient, requiring fewer lifting steps or chinese remaindering recoveries.

Fven if the unique solution is sought, the generalization presented here improves Wang's original problem, in the sense that more rational numbers may be reconstructed from the same residues modulo a. For example, if solutions to $\frac{\alpha}{\beta} \equiv 10 \bmod 21$ are to be found, Wang's algorithm returis nil, since for $\alpha, \beta<\lfloor\sqrt{12}\rfloor=3$ there are no solutions. Il, however; solutions to problem 2 with $c=5$ are sought, the pair $(\alpha, \beta)=(2,5)$ is returned by algorithm $\mathrm{R} \Lambda \mathrm{TCO}-$ NUN. The improvement is more significant when a bound $c$ for the denominator is known in advance.

\section{References}

[1] Beankamy, B., Trevisan. V. and Wang. P., Polynomial Factorizalion: Sharp Bounds, Efficient Algorithms, J. Symbolic Comp. 15., (1993), 393-413.

[2] Collins, G. E. and Encarnación, M. J., Effeient Rational Number Reconstruction, J. Symbolic Comp. 20, (1995), 287-297.

[3] Davenport, J. H., Sirct, Y. and Tournicr, E., Computer Algebra - Systems and Algorithms for Algcbraic Computation, Academic Press, (1988).

[4] Chistov, A. L., Grigoryev, D. Yu., Subexponential Time Solving Systems of Algebraic Fqualions I, Ussr Academy of Sciences, Steklov Mathematical Institute, Ieningrad Department, I OMI Preprints F-9-83, Leningrad 1983.

[5] Jacobs, D. P., Trevisan, V. and Wcbcr, K. E., Modular Sturm Sequences, LFRGS - CPGMAp Technical Report TR12-96 (http://www.mat.ufrgs.br).

[6] Sasaki, T. and Sasaki, M., On Inleger-lo-Ralional Conversion Algorilhm, SIGSAM Bulletin 26. No. 2, (1992), 19-21.

[7] Sorenson, J., T'wo f'ast GCD Algorithms, Journal of Algorithms 16, (1994), 110-144. 
[8] 'I'raverso, C., Ciröbner Irace Algorithms, Proc. of ISSAC'88, LNCS 358, Springer-Verlag; (1988), 125-138.

[9] Wang, P. S., A p-Adic Algorithm for Univariate Partial Fractions, Proccedings of 1981 ACM SYMSAC, 212-217.

[10] Wang, P. S., Guy, M. J. T. and Davemport, J. H., p-Adic Reconslaclion of Rational Numbers, SIGSAM Bulletin, Vol. 16. (1982), 2-3.

[11] Weber; K., The Acceleraled Inleger GCD Algorilhm, ACM TOMS 21, (1995), 111-122.

Technikum Joanneum

Fachhochschulgang Industrielle Filektronik

Werk VI Strasse 46

A-8605 Kaplenberg - Austria
UFRGS-Instituto de Matemática

Porto Alegre, RS

91509-900, Brazil

e-mail: trevisanomal.ufrgs.br 